\title{
Dynamic and structural properties of orthorhombic rare-earth manganites under high pressure
}

\author{
D. A. Mota, ${ }^{1}$ A. Almeida, ${ }^{1}$ V. H. Rodrigues,${ }^{2}$ M. M. R. Costa, ${ }^{2}$ P. Tavares,${ }^{3}$ P. Bouvier,${ }^{4}$ M. Guennou, ${ }^{5}$ \\ J. Kreisel, ${ }^{5,6}$ and J. Agostinho Moreira ${ }^{1, *}$ \\ ${ }^{1}$ Instituto de Física dos Materiais da Universidade do Porto and Institute of Nanoscience and Nanotechnology, Departamento de Física e \\ Astronomia da Faculdade de Ciências, Universidade do Porto, Rua do Campo Alegre, 687, 4169-007 Porto, Portugal \\ ${ }^{2}$ Centro de Estudos de Materiais por Difração de Raios X, Departamento de Física, Faculdade de Ciências e Tecnologia, Universidade de \\ Coimbra, 3004-516 Coimbra, Portugal \\ ${ }^{3}$ Centro de Química-Vila Real, Departamento de Química. Universidade de Trás-os-Montes e Alto Douro, 5000-801 Vila Real, Portugal \\ ${ }^{4}$ Laboratoire des Matériaux et du Génie Physique, Centre National de la Recherche Scientifique, Université Grenoble-Alpes, 3 parvis Louis \\ Néel, 38016 Grenoble, France \\ ${ }^{5}$ Département Science et Analyse des Matériaux, Centre de Recherche Publique Gabriel Lippmann, 41 Rue du Brill, L-4422 Belvaux, \\ Luxembourg \\ ${ }^{6}$ Physics and Materials Science Research Unit, University of Luxembourg, 41 Rue du Brill, L-4422 Belvaux, Luxembourg
}

(Received 27 February 2014; published 8 August 2014)

\begin{abstract}
We report a high-pressure study of orthorhombic rare-earth manganites $A \mathrm{MnO}_{3}$ using Raman scattering (for $A=\mathrm{Pr}, \mathrm{Nd}, \mathrm{Sm}, \mathrm{Eu}, \mathrm{Tb}$, and Dy) and synchrotron x-ray diffraction (XRD), for $A=\mathrm{Pr}, \mathrm{Sm}, \mathrm{Eu}$, and Dy. In all cases, a phase transition was evidenced by the disappearance of the Raman signal at a critical pressure that depends on the $A$ cation. For the compounds with $A=\mathrm{Pr}, \mathrm{Sm}$, and Dy, XRD confirms the presence of a corresponding structural transition to a noncubic phase, so that the disappearance of the Raman spectrum can be interpreted as an insulator-to-metal transition. We analyze the compression mechanisms at work in the different manganites via the pressure dependence of the lattice parameters, the shear strain in the $a c$ plane, and the Raman bands associated with out-of-phase $\mathrm{MnO}_{6}$ rotations and in-plane $\mathrm{O} 2$ symmetric stretching modes. Our data show a crossover across the rare-earth series between two different kinds of behavior. For the smaller $A$ cations considered in this study (Dy and $\mathrm{Tb}$ ), the compression is nearly isotropic in the $a c$ plane, with only small evolutions of the tilt angles and cooperative Jahn-Teller distortion. As the radius of the $A$ cation increases, the pressure-induced reduction of Jahn-Teller distortion becomes more pronounced and increasingly significant as a compression mechanism, while the pressure-induced tilting of octahedra chains becomes conversely less pronounced. We finally discuss our results in light of the notion of chemical pressure and show that the analogy with hydrostatic pressure works quite well for manganites with the smaller $A$ cations considered in this paper but can be misleading with large $A$ cations.
\end{abstract}

DOI: 10.1103/PhysRevB.90.054104

PACS number(s): 78.30.-j, 62.50.-p, 61.05.cp, 75.47.Lx

\section{INTRODUCTION}

The transition metal oxides with perovskite structure have attracted a huge interest due to their remarkable functional properties: ferroelectricity, superconductivity, magnetoresistivity, magnetism, multiferroicity, among others [1]. This versatility finds its origin in the couplings and competitions between different phenomena with similar energies (high polarizability of the crystal lattice, and strong electronic correlations). This gives rise to different phases whose stabilization strongly depends on external parameters like temperature, magnetic/electric field, chemical substitution, strain, and hydrostatic pressure. Among the transition metal oxides, the rare-earth manganites $A \mathrm{MnO}_{3}$ have been extensively studied. These compounds crystallize at ambient conditions into the Pnma structure [2]. The main distortions in orthorhombic manganites are the collective rotations (tilts) of the $\mathrm{MnO}_{6}$ octahedra with $a^{-} b^{+} a^{-}$tilting scheme in Glazer's notation and a cooperative Jahn-Teller distortion (CJTD) with orbital ordering. The structural distortions are intimately linked to the physical properties. It is notably well established that the balance between the competitive antiferromagnetic and

\footnotetext{
*jamoreir@fc.up.pt
}

ferromagnetic interactions in rare-earth manganites can be changed by varying the tilt of the $\mathrm{MnO}_{6}$ octahedra, pointing to a strong spin-lattice coupling [3,4]. Much work has therefore been devoted to the fine tuning of these distortions by cation substitution or external parameters.

Among the possible external parameters, hydrostatic pressure has such specificity that it allows modifying the interatomic distances, and thus the interactions, to a much larger extent than any other parameter like temperature, epitaxial strain, or magnetic field. However, high-pressure studies on manganites are still relatively scarce. The emphasis has been mostly laid on the model compound $\mathrm{LaMnO}_{3}$. A first report by Loa et al. [5] of the suppression of the Jahn-Teller distortion at $18 \mathrm{GPa}$ followed by an insulator-to-metal transition at $32 \mathrm{GPa}$ triggered a number of theoretical studies devoted to Jahn-Teller distortion under pressure and the mechanism responsible for the insulator-to-metal transition [6-9]. Experimentally, subsequent Raman and extended x-ray absorption fine structure (EXAFS) studies have given evidence for the persistence of the Jahn-Teller distortion over the entire range of the insulating phase at the local scale $[10,11]$. In contrast, only a couple of works have been reported on other manganites. Combined $\mathrm{x}$-ray diffraction and x-ray absorption spectroscopy studies on $\mathrm{TbMnO}_{3}$ and $\mathrm{DyMnO}_{3}$ showed a gradual decrease of the Jahn-Teller distortion and a bandwidth broadening, but 
could not clarify the presence of insulator-to-metal transition itself $[12,13]$. High-pressure Raman studies of $\mathrm{TbMnO}_{3}$ and $\mathrm{PrMnO}_{3}$ up to $10 \mathrm{GPa}$ [14] were focused on the compression mechanisms as inferred from the pressure evolution of Raman wave numbers. Recently, we reported synchrotron $\mathrm{x}$-ray diffraction and Raman spectroscopy studies of $\mathrm{GdMnO}_{3}$ under pressure up to $55 \mathrm{GPa}$ and showed evidence for a first-order structural transition accompanied by an insulator-to-metal transition around $50 \mathrm{GPa}$ [15]. In contrast, Muthu et al. [16] have not observed any structural transition at all up to $50 \mathrm{GPa}$ for $A=\mathrm{Eu}, \mathrm{Gd}, \mathrm{Tb}$, and $\mathrm{Dy}$.

In spite of these efforts, a broad overview of the effect of hydrostatic pressure on rare-earth manganites is still lacking, and open questions remain, notably regarding the persistence or disappearance of the Jahn-Teller distortion at high pressure and the existence of insulator-to-metal or structural transitions. The aim of this paper is to address those questions by a systematic study of a large set of orthorhombic rare-earth manganites (from $\mathrm{PrMnO}_{3}$ to $\mathrm{DyMnO}_{3}$ ). Using Raman spectroscopy and $\mathrm{X}$-ray powder diffraction (XRD) at room temperature up to the $55 \mathrm{GPa}$ range, we identify the effect of the $A$ cation radius on the compression mechanisms, we give evidence for the occurrence of an insulator-to-metal and structural transitions in all compounds, and discuss the evolution of octahedra tilts and Jahn-Teller distortion. The data presented here allow us to compare the effect of hydrostatic pressure and "chemical pressure" induced by changing the rare-earth cation radius.

\section{EXPERIMENTAL DETAILS}

High-quality ceramic $A \mathrm{MnO}_{3}(A=\mathrm{Pr}, \mathrm{Nd}, \mathrm{Sm}, \mathrm{Eu}, \mathrm{Tb}$, and Dy) samples were prepared with the sol-gel urea combustion method [17], and its chemical, morphological, and structural characteristics were checked by powder XRD, scanning electron microscopy (SEM), energy dispersive spectroscopy (EDS), and x-ray photoelectron spectroscopy (XPS). All highpressure experiments were carried out at room temperature. The ceramics were gently ground in ethanol to obtain fine powder samples of $A \mathrm{MnO}_{3}$ which were loaded in a diamond anvil cell (DAC) with diamond culets of $300 \mu \mathrm{m}$ diameter and with helium as a pressure-transmitting medium. Although helium crystallizes at $12 \mathrm{GPa}$ at ambient temperature, it is found to provide quasihydrostatic conditions up to $50 \mathrm{GPa}$ with standard deviation in pressure measurements of only $0.3-0.5 \%$ at $150 \mathrm{GPa}[18,19]$. The pressure inside the pressure chamber was measured by the standard ruby fluorescence method [20]. We have monitored the pressure value before and after each data acquisition, and the difference between the obtained values is less than $0.1 \mathrm{GPa}$ up to $55 \mathrm{GPa}$. The Raman spectra were recorded on a LabRam spectrometer using a He-Ne laser at $633 \mathrm{~nm}$. The laser power was kept below $5 \mathrm{~mW}$ on the DAC to avoid sample heating. The spectra were fitted with a sum of independent damped oscillators on top of a polynomial baseline. High-pressure synchrotron XRD experiments were performed on the Extreme Conditions Beamline (ECB) at the Positron-Electron Tandem Ring Accelerator (PETRA) III, Deutsches Elektronen-Synchrotron (DESY), and at the European Synchrotron Radiation Facility (ESRF) on the ID27 high-pressure beamline. X-ray diffraction patterns were collected on a Perkin Elmer image detector at wavelength $\lambda=$
$0.29118 \AA$ for $\mathrm{PrMnO}_{3}$ and $\mathrm{SmMnO}_{3}$ (PETRA III) and on a Mar charge-coupled device (CCD) detector at $\lambda=0.3738 \AA$ for $\mathrm{EuMnO}_{3}$ and $\mathrm{DyMnO}_{3}$ (ID27). Diffraction patterns were obtained after integration of raw data using FIT2D [21]. The powder diffraction data were analyzed by Le Bail refinements using FullProf software [22]. The refined parameters were the lattice parameters, the parameters of a pseudo-Voigt profile and the zero shift. The XRD spectra background was fitted by linear interpolation between a set of manually chosen points for each diffractogram. The coordinates of these points were not refined.

\section{EXPERIMENTAL RESULTS}

\section{A. High-pressure x-ray diffraction}

Figure 1 shows representative XRD patterns of $A \mathrm{MnO}_{3}$ compounds, with $A=\mathrm{Pr}, \mathrm{Sm}, \mathrm{Eu}, \mathrm{Dy}$, recorded at different pressures.

For all studied compounds, the characteristic Pnma XRD pattern can be followed at low pressures, with pressureinduced shifts of the Bragg peaks towards larger $2 \theta$ due to the volume reduction. For EuMnO 3 , patterns collected up to $38.8 \mathrm{GPa}$ exhibit a marked broadening of the diffraction peaks but no sign of a phase transition. In all other cases (Pr, Sm, Dy), a phase transition is revealed by changes in the diffraction patterns, at a transition pressure (hereafter designated by $P_{\mathrm{IM}}$ ) whose value strongly depends on the rare-earth element. In the three cases, we observe a coexistence of the low-pressure and high-pressure phases, and a hysteresis behavior upon decreasing pressure, which is indicative of the first-order nature of the structural phase transitions.

Possible structures for high-pressure phases of $\mathrm{PrMnO}_{3}$, $\mathrm{SmMnO}_{3}$, and $\mathrm{DyMnO}_{3}$ were tested by profile matching. For $\mathrm{PrMnO}_{3}$, a good profile matching accounting for all observed diffraction peaks was obtained with a tetragonal cell with space group $I 4 / \mathrm{mcm}$ and $a=5.078(3) \AA$ and $c=$ $7.442(5) \AA$, at $42.7 \mathrm{GPa}$. This space group is very common in perovskites and corresponds to the $\mathrm{a}^{o} \mathrm{a}^{o} \mathrm{c}^{-}$tilt system. $\mathrm{SmMnO}_{3}$ gave less convincing results due to the limited quality of the diffraction diagrams and the width of diffraction peaks, and it was not possible to find a suitable space group. $\mathrm{DyMnO}_{3}$ is a special case: the transition to the high-pressure phase is not revealed by emergence or disappearance of diffraction peaks but rather by abrupt shifts of existing peaks, and we find that the XRD pattern at high pressure remains compatible with the Pnma symmetry, while all other trials resulted in poorer profile refinements. We therefore hypothesize that $\mathrm{DyMnO}_{3}$ undergoes an isosymmetric transition. Although such transitions are admittedly quite rare, we note that similar Pnma $\rightarrow$ Pnma transitions have already been reported in orthoferrites under pressure in a similar pressure range [23].

Even though the information regarding the crystal structures above the transition remains incomplete and uncertain, the evidence for phase transitions is very clear and contradicts the absence of phase transitions reported in Ref. [16]. This can most probably be accounted for by the pressure-transmitting medium used in that study (ethanol-methanol instead of helium), which produces very strong deviatoric stress above 
$10 \mathrm{GPa}$. Besides, we note that, remarkably, no common rule seems to emerge from these preliminary results. In fact, the high-pressure phases proposed so far are very different and even belong to different crystal systems $\left(\mathrm{GdMnO}_{3}\right.$ : cubic $P 2_{1} 3$ [15], $\mathrm{PrMnO}_{3}$ : tetragonal I4/mcm; $\mathrm{DyMnO}_{3}$ : orthorhombic Pnma). $\mathrm{BiMnO}_{3}$ even exhibits an intermediate monoclinic polar phase between the insulating Pnma phase and the high-pressure metallic phase [24]. Last, even if helium provides very good hydrostatic conditions up to this pressure range, stress may develop between grains in a powder and cause shifts of transition pressures or prevent the determination of the high-pressure structures. Single crystal studies would be necessary to conclusively waive this problem.

In Fig. 2, we show the pressure dependence of the pseudocubic lattice parameters defined as $a_{p c}=a / \sqrt{ } 2, b_{p c}=b / 2$,
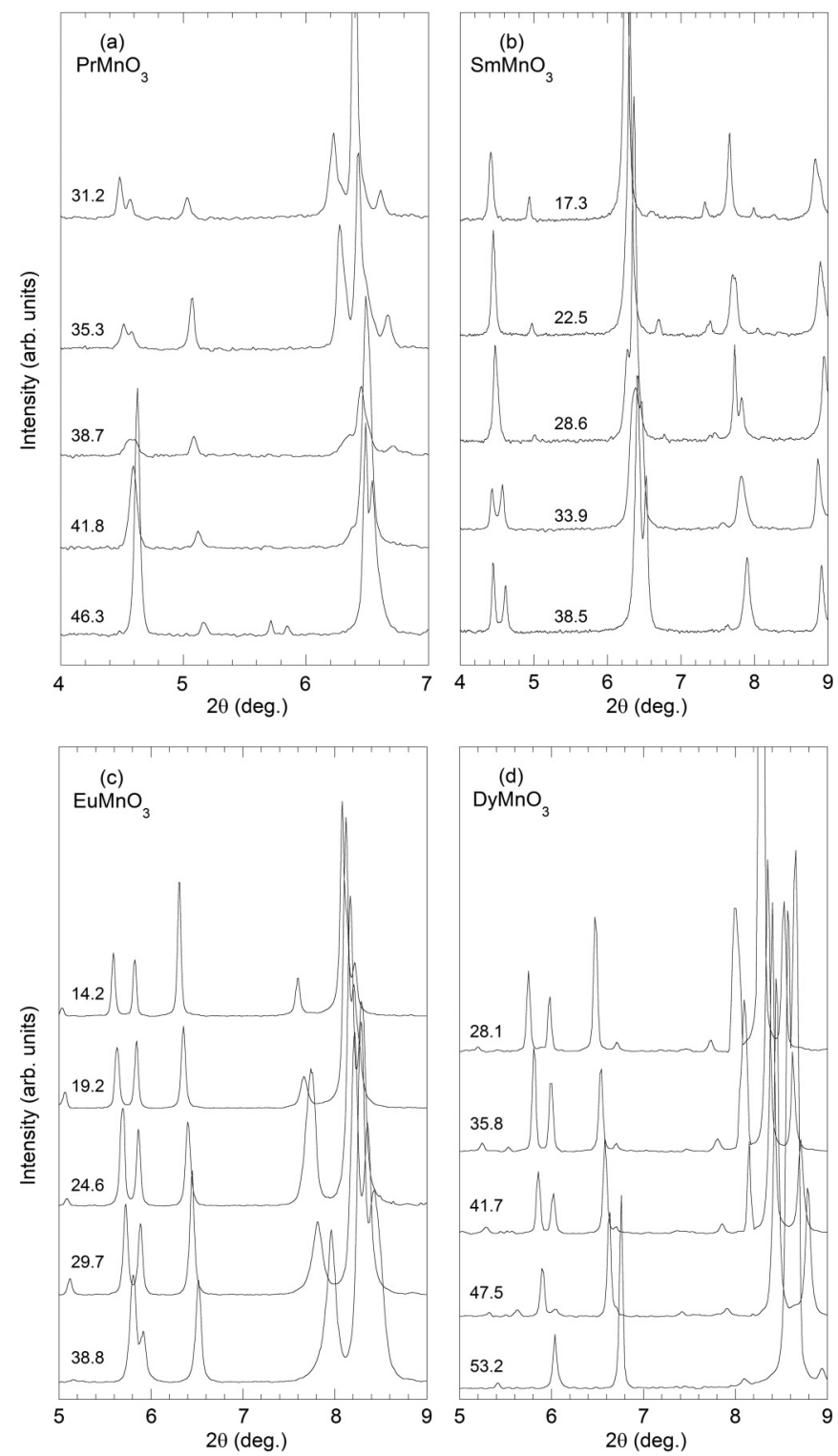

FIG. 1. XRD patterns of (a) $\mathrm{PrMnO}_{3}$ and (b) $\mathrm{SmMnO}_{3}$, recorded at PETRA III $(\lambda=0.29118 \AA),\left(\right.$ c) $\mathrm{EuMnO}_{3}$, and (d) $\mathrm{DyMnO}_{3}$, recorded at ID27 $(\lambda=0.3738 \AA$ ) at selected pressures (expressed in $\mathrm{GPa}$ ).

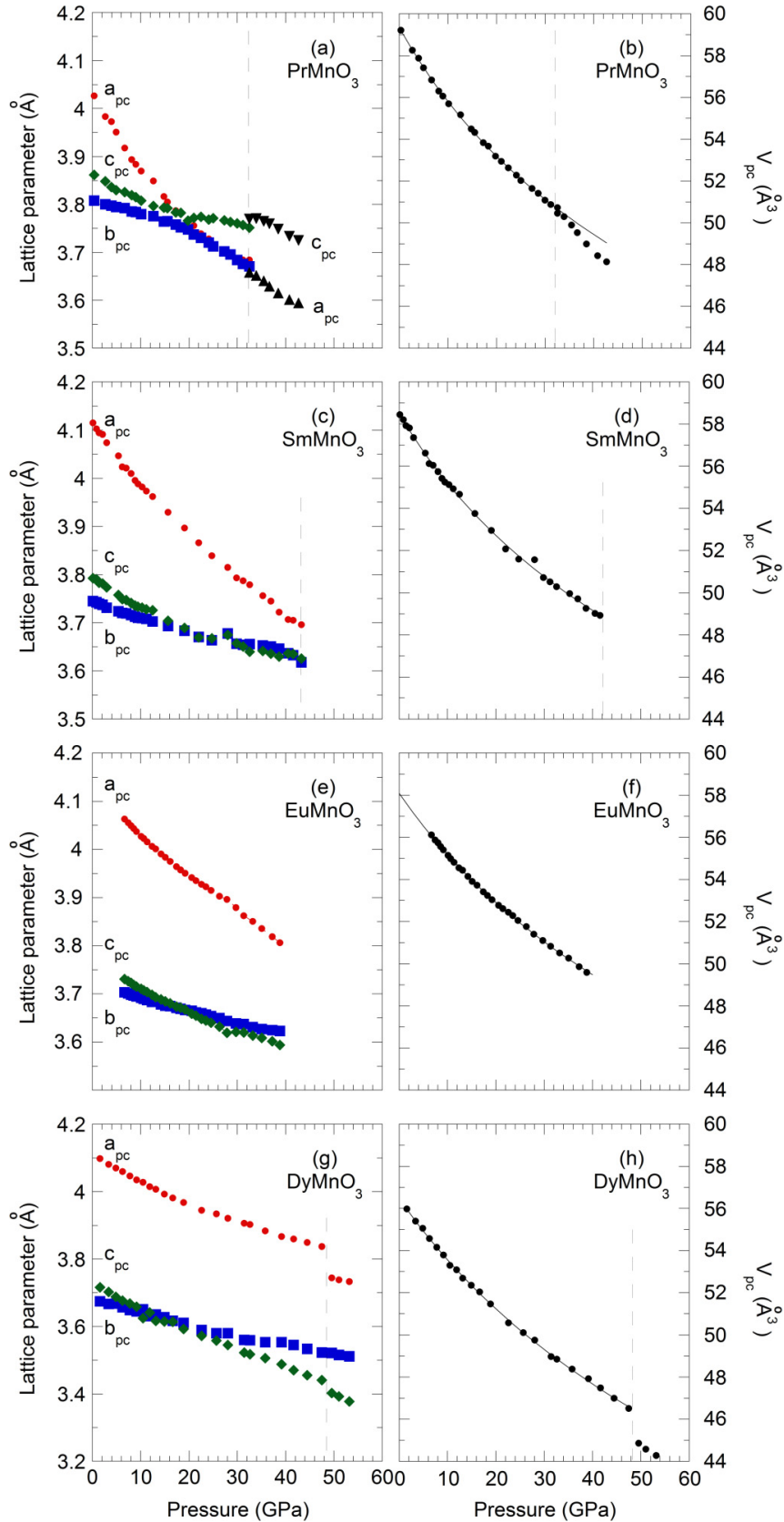

FIG. 2. (Color online) Pressure dependence of the pseudocubic lattice parameters and volume of $\mathrm{PrMnO}_{3}, \mathrm{SmMnO}_{3}, \mathrm{EuMnO}_{3}$, and $\mathrm{DyMnO}_{3}$ : red circles $a_{p c}$, green diamonds $c_{p c}$, blue squares $b_{p c}$. The vertical dashed lines mark the transition pressure. The solid lines in the volume plots are the best fits to the Birch-Murnaghan equation of state [see Eq. (1)]. For $\mathrm{SmMnO}_{3}$, whose high-pressure structure could not be unambiguously determined, we do not show the lattice parameters in the high-pressure phase.

and $c_{p c}=c / \sqrt{ } 2$, and of the pseudocubic volume $V_{p c}$ (or volume per formula unit), defined as $V_{p c}=a b c / 4$. For $\mathrm{PrMnO}_{3}$ (respectively $\mathrm{DyMnO}_{3}$ ), the lattice parameters above $P_{\mathrm{IM}}$ are determined under the assumption of a tetragonal $I 4 / \mathrm{mcm}$ phase (respectively orthorhombic Pnma) with pseudocubic lattice parameters defined as $a_{p c}=a / \sqrt{ } 2$ and $c_{p c}=c / 2$. For $\mathrm{SmMnO}_{3}$, whose high-pressure structure could not be 
TABLE I. Values of the bulk modulus $B_{0}$ and its pressure derivative $B_{o}^{\prime}$, obtained from the best fit of the third-order Birch-Murnaghan equation of state to the pseudocubic volume. The corresponding values of $\mathrm{LaMnO}_{3}$ were taken from Ref. [5].

\begin{tabular}{lrcc}
\hline \hline & $B_{0}(\mathrm{GPa})$ & $B_{o}^{\prime}$ & Reference \\
\hline $\mathrm{LaMnO}_{3}$ & $97 \pm 2$ & $9.7 \pm 0.4$ & {$[5]$} \\
$\mathrm{PrMnO}_{3}$ & $139 \pm 4$ & $4.9 \pm 0.5$ & This paper \\
$\mathrm{SmMnO}_{3}$ & $150 \pm 8$ & $4.9 \pm 0.3$ & This paper \\
$\mathrm{EuMnO}_{3}$ & $172 \pm 5$ & $4.6 \pm 0.3$ & This paper \\
$\mathrm{GdMnO}_{3}$ & $170 \pm 1$ & $3.72 \pm 0.07$ & This paper \\
$\mathrm{DyMnO}_{3}$ & $171 \pm 9$ & $3.8 \pm 0.3$ & This paper \\
\hline \hline
\end{tabular}

determined, we do not show lattice parameters in the highpressure phase. The quality of the data precluded meaningful Rietveld refinements, so that a detailed analysis of the relevant bond lengths and angles was not possible. In the orthorhombic phase, the pressure dependence of the unit cell volume of all studied compounds could be modeled adequately by a third-order Birch-Murnaghan equation of state [25]

$$
P(V)=3 B_{0} f_{E}\left[1+2 f_{E}\right]^{5 / 2}\left\{1+\frac{3}{4}\left(B_{o}^{\prime}-4\right) f_{E}\right\},
$$

where $B_{0}$ is the bulk modulus and $B_{o}^{\prime}$ its first pressure derivative, all taken at ambient pressure, and

$$
f_{E}=\frac{1}{2}\left\{\left[\frac{V_{p c}(0)}{V_{p c}}\right]^{2 / 3}-1\right\} .
$$

In Eq. (2), $V_{p c}(0)$ is the pseudocubic volume at room pressure, and $V_{p c}$ is the pseudocubic volume at pressure $P$.

Figure 2(h) shows a $4 \%$ volume drop at the transition in $\mathrm{DyMnO}_{3}$ associated with the first-order character of the transition. Such a volume decrease was also reported for $\mathrm{GdMnO}_{3}$ [15]. In $\mathrm{PrMnO}_{3}$, a volume drop is not observed, but the coexistence of phases and the absence of a group/subgroup relation between the high-pressure phase and the low-pressure phase are nonetheless indications of the first-order nature of the transition.

Table I presents the values of $B_{0}$ and $B_{o}^{\prime}$ obtained from the fit of the third-order Birch-Murnaghan equation of state [Eq. (1)] to the pseudocubic volume. Table I also shows the $B_{0}$ and $B_{o}^{\prime}$ values, taken from Ref. [5]. The bulk modulus increases as the $A$ cation size decreases from $\mathrm{La}$ to $\mathrm{Eu}$ and then remains constant for Eu-Dy. We note that the increase of the bulk modulus with decreasing $A$ cation size has been also found in rare-earth chromites $\mathrm{SmCrO}_{3}, \mathrm{EuCrO}_{3}$, and $\mathrm{GdCrO}_{3}$ [26]. Moreover, the pressure derivative of the bulk modulus decreases with decreasing the $A$ cation size, up to $\mathrm{GdMnO}_{3}$, where the minimum value is reached. It is worth stressing that the $B_{o}^{\prime}$ value reported for $\mathrm{LaMnO}_{3}$ is unusually high, far above the values obtained for the other rare-earth manganites or similar perovskites [5].

\section{B. Raman spectroscopy}

Before going into the details of high-pressure data, we want to recall a few facts about the Raman spectra of manganites at ambient conditions. The Raman activity in rare-earth manganites arises from structural deviations from the ideal cubic perovskite symmetry. The basic distortions involved in symmetry reduction from $P m \overline{3} m$ to Pnma are the [010] and [101] rotations of the $\mathrm{MnO}_{6}$ octahedra. Moreover, as the $\mathrm{Mn}^{3+}$ ion is Jahn-Teller active, a CJTD occurs, but does not lower the symmetry any further. Due to octahedral tilting and CJTD, the rare-earth ion ( $A$ cation) is displaced from the

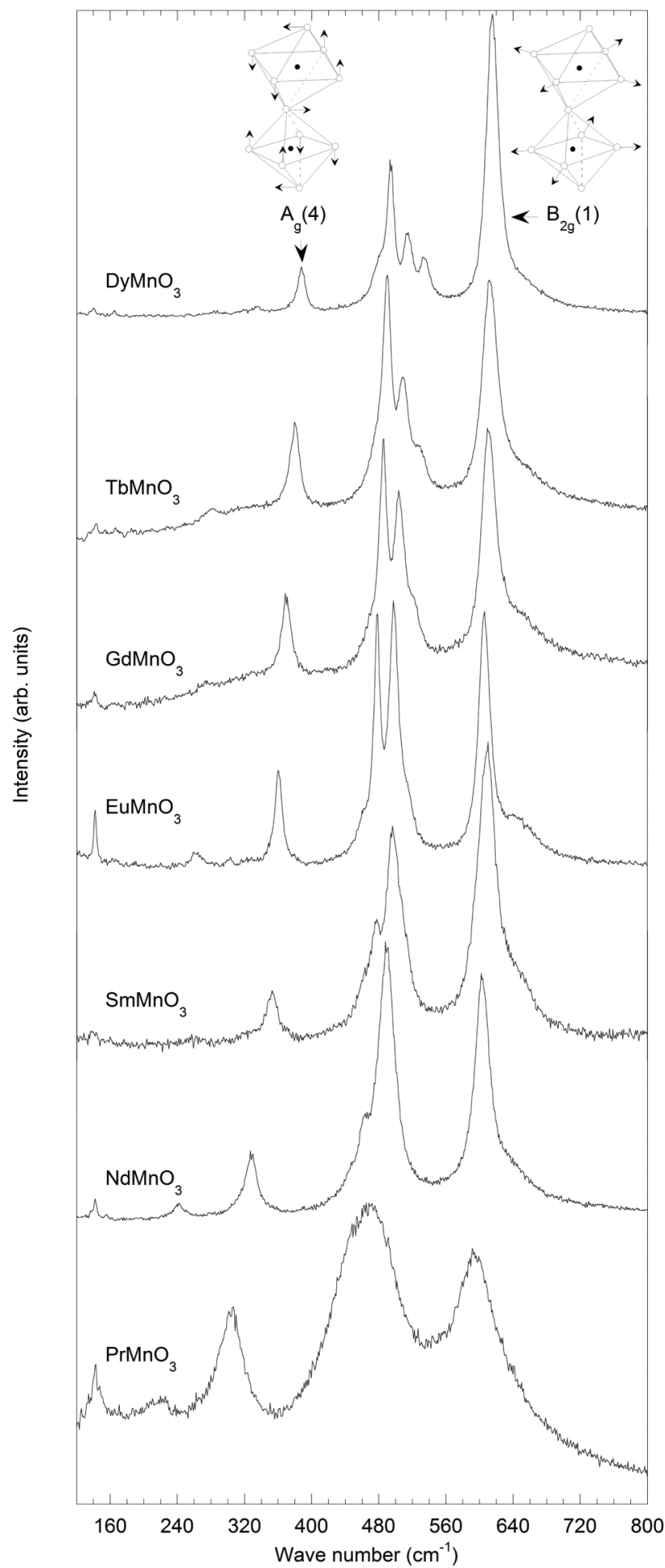

FIG. 3. Unpolarized Raman spectra of the $A \mathrm{MnO}_{3}$ compounds at ambient conditions. The out-of-phase $\mathrm{MnO}_{6}$ rotation mode $\left[A_{g}(4)\right]$ and the in-plane $\mathrm{O} 2$ symmetric stretching mode $\left[B_{2 g}(1)\right]$ are labeled in this figure. The sketches show the atomic motions involved in these modes. 
high-symmetry position (at the center of the eight surrounding $\mathrm{MnO}_{6}$ octahedra), and thus its coordination reduces from 12 to 8 , but it remains in the mirror plane. The octahedral rotations cause the equatorial long $\mathrm{Mn}-\mathrm{O}$ bonds to align more closely with the $a$ axis than with the $c$ axis. Due to these distortions, the four oxygen atoms belonging to the equatorial plane of the $\mathrm{MnO}_{6}$ octahedra (hereafter designated by O2) occupy general positions, while the $\mathrm{Mn}^{3+}$ ion remains on the inversion center and the apical oxygen atoms (designated by $\mathrm{O} 1$ ) remain in the mirror plane. Factor group analysis provides the following decomposition corresponding to the 60 normal vibrations at the $\Gamma$ point of the Brillouin zone

$$
\begin{aligned}
\Gamma_{\text {acoustic }}= & B_{1 u}+B_{2 u}+B_{3 u}, \\
\Gamma_{\text {optical }}= & \left(7 A_{g}+5 B_{1 g}+7 B_{2 g}+5 B_{3 g}\right)_{\text {Raman-active }} \\
& +\left(9 B_{1 u}+7 B_{2 u}+9 B_{3 u}\right)_{\mathrm{IR}-\text { active }}+\left(8 A_{u}\right)_{\text {silent }} .
\end{aligned}
$$

Figure 3 shows the unpolarized Raman spectra of $A \mathrm{MnO}_{3}$, with $A=\mathrm{Pr}, \mathrm{Nd}, \mathrm{Sm}, \mathrm{Eu}, \mathrm{Tb}$, and Dy, recorded at ambient conditions. Due to the polycrystalline nature of the studied samples, the Raman spectra exhibit simultaneously all Ramanactive modes $A_{g}, B_{1 g}, B_{2 g}$, and $B_{3 g}$. The Raman peaks are
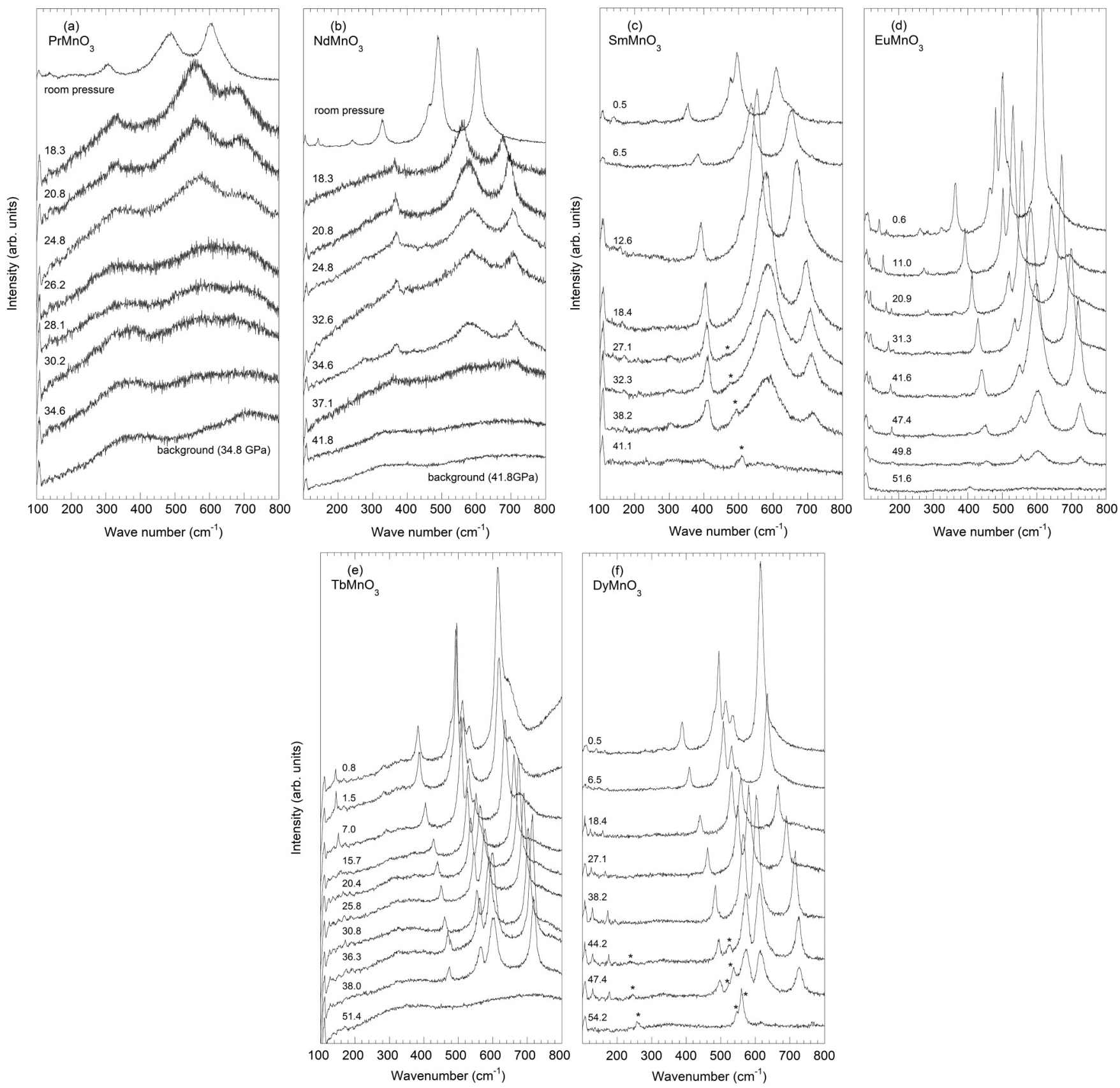

FIG. 4. Raman spectra of the $A \mathrm{MnO}_{3}$ compounds, recorded at room temperature and at fixed hydrostatic pressure (expressed in GPa). The bands marked with asterisks correspond to Raman modes arising from $\mathrm{N}_{2}$ and $\mathrm{O}_{2}$ solid phases, which were unintentionally introduced as a minor phase in a helium transmitting medium in the DAC preparation procedure. For the $\mathrm{PrMnO}_{3}$ and $\mathrm{NdMnO}_{3}$ cases, a background Raman spectrum measured next to the sample in the pressure transmitting medium is also shown for comparison. 
more clearly separated as the $A$ cation size decreases, due to the corresponding increase of the orthorhombic distortion. Our Raman spectra are in excellent agreement with those previously reported in the literature [27,28], and we shall use throughout the paper the mode assignment and the notations defined in Ref. [28]. In the following, we shall focus our attention on the Raman bands whose activation is associated with the main structural deformations allowing for symmetry reduction, namely, the out-of-phase $\mathrm{MnO}_{6}$ rotation mode, with $A_{g}$ symmetry, activated by the [101] rotations, and the in-plane $\mathrm{O} 2$ symmetric stretching mode, with $B_{2 g}$ symmetry, activated by the Jahn-Teller distortion [28]. These bands will be further used to monitor the pressure-induced deformations in the $\mathrm{AMnO}_{3}$ compounds. We have labeled these bands in Fig. 3, accompanied by a sketch of the modes, showing the atomic displacements associated with each mode. It is well known that the wave number of the in-plane $\mathrm{O} 2$ symmetric stretching mode involves stretching vibrations of the equatorial plane $\mathrm{O} 2$ atoms, whose wave number is determined by the $\mathrm{Mn}-\mathrm{O} 2$ distances $[28,29]$. On the other hand, the wave number of the out-of-phase $\mathrm{MnO}_{6}$ rotation mode scales with the tilt angle [28,29].

Figure 4 displays representative Raman spectra recorded at selected hydrostatic pressures. As pressure increases, the Raman bands shift towards higher wave numbers due to an overall pressure-induced bond shortening and volume reduction and become broader. The most notable result of Fig. 4 is the complete extinction of the Raman signature of all manganites above a critical pressure. At the highest pressure, a background Raman signal was recorded by focusing the laser in the pressure-transmitting medium next to the sample, in order to confirm that the remaining weak and broad bands do not originate from the sample. The interpretation of this change follows a reasoning already given for $\mathrm{LaMnO}_{3}, \mathrm{GdMnO}_{3}$, or $\mathrm{BiMnO}_{3}[5,10,15,24]$ and that we recall briefly here. Generally speaking, the vanishing of the Raman spectrum in a perovskite can be accounted for in two ways: (1) by a transition to the cubic phase where no Raman mode is allowed by symmetry, or (2) by a transition to a metallic state where the Raman signal is extremely weak due to the strong reduction of the penetration depth of the laser. For $\mathrm{PrMnO}_{3}$, $\mathrm{SmMnO}_{3}$, and $\mathrm{DyMnO}_{3}$, our XRD results clearly show that the structure in this pressure range is not cubic, so that we can discard the first option. For $\mathrm{NdMnO}_{3}, \mathrm{EuMnO}_{3}$, and $\mathrm{TbMnO}_{3}$, we do not have this evidence from XRD. Although further experimental studies are needed, it may be expected that they follow the same pattern. At this stage, our results enable us to conclude that all compounds undergo a structural phase transition, which is accompanied by an insulator-to-metal transition for the compounds with $A=\mathrm{Pr}, \mathrm{Sm}$, and Dy. The transition is reversible, and the Raman spectrum is recovered upon decreasing pressure with a significant hysteresis ranging between 3 to $6 \mathrm{GPa}$ depending on the rare-earth cation, corroborating the first-order nature of the high-pressure phase transition. Apart from this transition, no anomaly is detected in the spectra; the characteristic orthorhombic spectral profile is maintained up to the disappearance of the Raman signal, and no anomaly is detected in the pressure evolution of the Raman wave numbers (see Supplemental Material [30]), indicating that no change of symmetry occurs in the insulating phase.

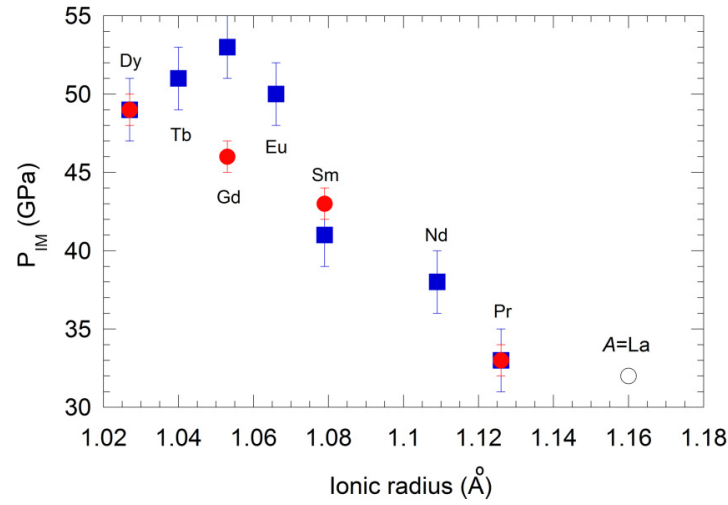

FIG. 5. (Color online) Critical pressures as a function of ionic radius determined from XRD patterns (circles) and Raman spectra (squares) upon increasing pressure. The value of transition pressure for $\mathrm{LaMnO}_{3}$ and $\mathrm{GdMnO}_{3}$ were obtained from Refs. [5,15], respectively.

Transition pressures determined from Raman and XRD data for all compounds are summarized in Fig. 5. Estimation of the critical pressure from the XRD data is in general more accurate than from the Raman data, since it is revealed by abrupt changes in the pressure dependence of the volume and lattice parameters. Determination of the transition pressure from the Raman spectra, on the other hand, requires determining the point where the spectrum vanishes, which is sometimes unclear when the signal is already weak with broad features. This could explain the large difference between the two techniques for $\mathrm{GdMnO}_{3}$ [15]. The transition pressure tends to increase for smaller cations, with a possible stabilization at approximately $50 \mathrm{GPa}$ for Eu-Dy.

\section{DISCUSSION}

\section{A. Compression mechanisms and evolution of tilts}

In this section, we focus on the evolution of elementary structural distortions and compression mechanisms in the investigated manganites. We first consider the evolution of tilts, which can be done in general from the evolution of the corresponding Raman bands, namely the $A_{g}(2)$ and $A_{g}(4)$ modes. For all $\mathrm{AMnO}_{3}$ compounds, the $A_{g}(4)$ mode is well defined and could be followed nicely with pressure, but the $A_{g}(2)$ is much more difficult to follow for two reasons: first, because it can mix with the $A_{g}(7) A$-shift mode depending on the $A$ cation size [28], and second, because it is hardly seen for large rare-earth for which the orthorhombic distortion is small and, consequently, the Raman spectrum is broad and weak in intensity. Nonetheless, for $\mathrm{GdMnO}_{3}$ [15], we have measured pressure variation of $2.47 \mathrm{~cm}^{-1} / \mathrm{GPa}$ for the $A_{g}(4)$ tilt mode. If we consider that this mode has the same linear dependence with the tilt angles of $23.5 \mathrm{~cm}^{-1} / \mathrm{deg}$, as demonstrated by Iliev [28], we can expect pressure variation of $0.105^{\circ} / \mathrm{GPa}$ for the [101] tilt angle. Thus, we assume a tilt increase of $4.7^{\circ}$ $(28 \%)$ for the [101] tilt angle, for a pressure increase of $45 \mathrm{GPa}$ in $\mathrm{GdMnO}_{3}$. As discussed before, the tilt increase is even lower for large $A$ cations such as $\mathrm{PrMnO}_{3}$ for which we can extract a pressure variations of $0.059^{\circ} / \mathrm{GPa}$ or a tilt increase of $1.9^{\circ}$ (15\%) for the [101] tilt angle for a pressure increase of $32 \mathrm{GPa}$. 

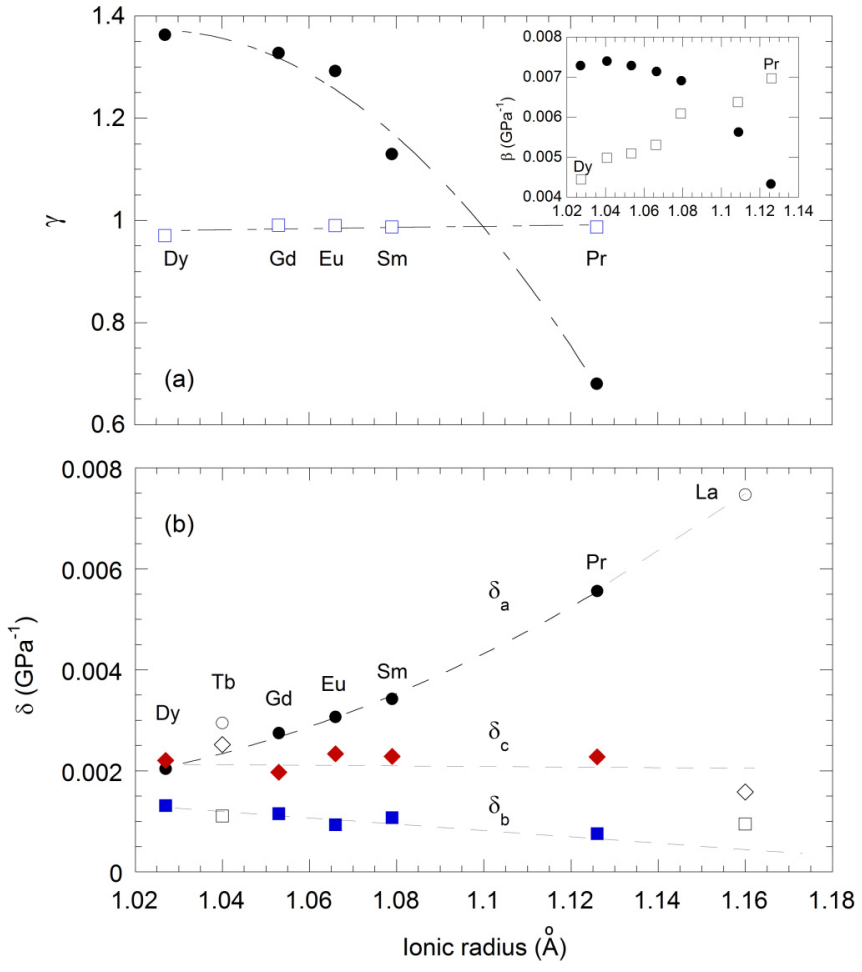

FIG. 6. (Color online) (a) Mode Grüneisen parameter $\gamma$ of the out-of-phase $\mathrm{MnO}_{6}$ rotations $A_{g}(4)$ (circles) and of the in-plane $\mathrm{O} 2$ symmetric stretching $B_{2 g}(1)$ (squares) modes. The inset depicts the coefficient $\beta$, defined by Eq. (4). (b) Compressibility of the lattice parameters as a function of the $A$ cation size: $\delta_{a}$ circles, $\delta_{b}$ squares, and $\delta_{c}$ diamonds. Closed symbols are our data; open symbols for $\mathrm{LaMnO}_{3}$ and $\mathrm{TbMnO}_{3}$ were calculated from the data reported in Refs. [5,12], respectively. The dashed lines are guides for the eyes.

In order to relate directly the change of Raman wave number $\omega$ with the volume reduction, we show in Fig. 6(a) the mode Grüneisen parameter defined as [31,32]

$$
\gamma=\frac{d(\ln \omega)}{d\left(\ln V_{p c}\right)},
$$

where $\omega$ is the interpolated mode wave number at the same pressure of the obtained pseudocubic volume $V_{p c}$ value, and the $\gamma$ is the slope of the linear fit of the $\ln \omega$ versus $\ln V_{p c}$, over the full stability range of the Pnma phase. Additionally, we show in the insert the "mode compressibility" $\beta$ defined as

$$
\beta=\frac{1}{\omega_{0}}\left(\frac{d \omega}{d P}\right)_{o} .
$$

In Eq. (4), $\omega$ is the mode wave number at pressure $P$, and $\omega_{0}$ and $\left(\frac{d \omega}{d P}\right)_{o}$ are the mode wave number and its slope at room pressure, calculated from a linear fit in the 0-10 $\mathrm{GPa}$ pressure range, where the behavior can be roughly considered linear (see Supplementary Material [30]). Both quantities are given for the tilt mode $A_{g}(4)$ and the in-plane $\mathrm{O} 2$ symmetric stretching $B_{2 g}(1)$ modes, which probes the average $\mathrm{Mn}-\mathrm{O} 2$ bond distance. Remarkably, the latter has a Grüneisen parameter that is independent of the rare-earth cation, showing that the reduction of the average $\mathrm{Mn}-\mathrm{O} 2$ bond lengths plays an equal role for all studied compounds. In contrast, the former exhibits strong variations and changes by a factor of 2 between
DyMnO 3 and $\mathrm{PrMnO}_{3}$. The mode compressibilities $\beta$ for the two Raman modes show a similar crossover. Considering the mode Grüneisen parameter (or $\beta$ ) as a measure of the role of the distortion in the volume reduction, we conclude that the change in the tilt angle contributes much less to the volume reduction in compounds with large rare-earth cations and small tilt angles (La-Pr) than in compounds with smaller rare-earth cations and larger tilt angles (Dy-Sm).

\section{B. Jahn-Teller distortion: spontaneous strain and Raman spectroscopy}

We then move to the discussion of the pressure-evolution of the CJTD. To this end, we first show in Fig. 6(b) linear compressibilities $\delta_{u}(u=a, b, c)$ determined by a least-square fit of the lattice parameters for $P<P_{I M}$ against a third-order Birch-Murnaghan equation of state $\left[\delta_{u}=1 /\left(3 B_{o}\right)\right][33,34]$. In $\mathrm{LaMnO}_{3}$, the compression is strongly anisotropic, but the anisotropy gradually decreases for smaller cations and practically vanishes in $\mathrm{DyMnO}_{3}$. In all cases, the hardest direction is along the $b$ axis, and $\delta_{b}$ exhibits a slight decrease for the larger ionic radii. Here, $\delta_{c}$ is nearly independent of the rare-earth element, while $\delta_{a}$ shows the strongest variations. This anisotropy of the compressibility with $\delta_{a}>\delta_{c}>\delta_{b}$ therefore exactly counteracts the effects of the CJTD, namely a strong elongation of $a$ and a moderate contraction of $b$ while $c$ remains nearly unchanged [35]. Let us recall that the long and short $\mathrm{Mn}-\mathrm{O} 2$ bonds have their main projections along the $a$ and $c$ axes, respectively. In addition, as the Jahn-Teller distortion leads to a volume increase of about $0.7 \%$ relative to the corresponding rare-earth ferrites with tilts and no Jahn-Teller distortion, its reduction does contribute to the compression of the crystal. Figure 6(b) shows that the Jahn-Teller reduction is more rapid with increasing pressure for compounds with large cations (La-Pr), but gets slower and slower as the ionic radius decreases. Ultimately, for $\mathrm{DyMnO}_{3}$, the nearly isotropic compression suggests that the Jahn-Teller distortion hardly changes. This is in good qualitative agreement with the (limited) data on $\mathrm{Mn}-\mathrm{O} 2$ bond lengths measured by Rietveld refinements for $\mathrm{LaMnO}_{3}, \mathrm{TbMnO}_{3}, \mathrm{DyMnO}_{3}$, and $\mathrm{GdMnO}_{3}[5,12,13,15]$.

This interpretation of the linear compressibilities can be complemented using the Landau formalism developed by Carpenter and Howard [36] and Denton and Ashcroft [37] for the structural evolution of perovskites containing Jahn-Teller cations and octahedral tilting. This approach gives a detailed account of the relationships between macroscopic strains and order parameters, in order to disentangle the combined effects of tilting and Jahn-Teller distortions in perovskites. For the Pnma perovskites, the total distortion involves three different order parameters. Two of them are associated with the out-of-phase rotations along $a$ and $c$ [order parameter labeled $q_{4}$, linked to the $A_{g}(4)$ Raman mode] and the in-phase rotation along $b\left[q_{2}\right.$, linked to $A_{g}(2)$ Raman mode], while the third one is associated with the Jahn-Teller distortion. All three order parameters couple with spontaneous strains, the shear strain $e_{4}$ being the most insightful component to follow for a discussion of Jahn-Teller distortion due to its simpler dependence on the order parameters. If the tilt angles show only small changes, a complete suppression of the Jahn-Teller distortion $\left(q_{2 J T}=0\right)$ 


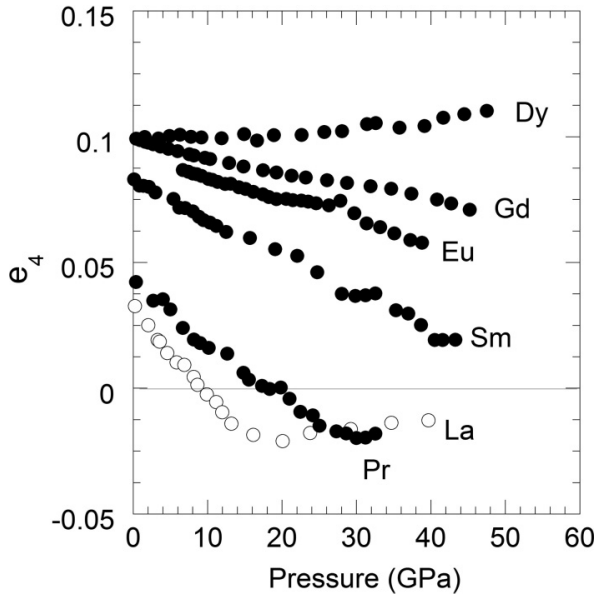

FIG. 7. Pressure dependence of the shear strain $e_{4}$ for $A \mathrm{MnO}_{3}$, with $A=\mathrm{La}, \mathrm{Pr}, \mathrm{Sm}, \mathrm{Eu}, \mathrm{Gd}, \mathrm{Tb}$, and Dy. Data for $\mathrm{LaMnO}_{3}$ and $\mathrm{GdMnO}_{3}$ were taken from Refs. [5,15], respectively.

should be detected as an anomaly in the pressure evolution of the shear strain, as it was claimed in Ref. [37] for $\mathrm{LaMnO}_{3}$ on the basis of the experimental XRD data from Ref. [5].

In the following, we will carry out a similar analysis for our series of compounds. Like in Ref. [37], the reference state is defined by the axes of the simple cubic perovskite cell and the shear strain is defined as

$$
2 e_{23}=e_{4}=\frac{a_{p c}-c_{p c}}{a_{o}},
$$

with a reference cubic parameter $a_{0}$ given by

$$
a_{o}=\left(a_{p c} \times b_{p c} \times c_{p c}\right)^{1 / 3} \text {. }
$$

In principle, the value of $a_{0}$ should be calculated from an extrapolation of the real evolution of the lattice parameter in the cubic phase, but no such data is available. This definition, which implicitly assumes that the non-symmetry-breaking strain (or volume strain, usually written $e_{a}$ ) is zero, introduces a small error in the absolute value of the strains, of the order of the volume strain itself. However, this definition remains relevant for the search of anomalies in the evolution of the shear strain.

Figure 7 shows the pressure evolution of the shear strain $e_{4}$ for all the compounds studied in this work. We have also included the data of $\mathrm{LaMnO}_{3}$ and $\mathrm{GdMnO}_{3}$, obtained from the analysis of data reported in Refs. [5,15].

The shear strain $e_{4}$ is always positive at room pressure. Only for $\mathrm{LaMnO}_{3}$ and $\mathrm{PrMnO}_{3}$ does the shear strain exhibit an anomaly at around 16 and $30 \mathrm{GPa}$, respectively. For $\mathrm{SmMnO}_{3}$, $\mathrm{EuMnO}_{3}$, and $\mathrm{GdMnO}_{3}, e_{4}$ decreases with pressure but does not show any anomaly and remains always positive. Therefore, for $\mathrm{SmMnO}_{3}, \mathrm{EuMnO}_{3}, \mathrm{GdMnO}_{3}$, and $\mathrm{DyMnO}_{3}$, the strain analysis does not give any evidence for a suppression of the CJTD under pressure, and we may assume that it persists up to the transition pressure even on the average macroscopic scale.

Last, the average information given by XRD patterns can be compared to the Raman spectroscopy results. The wave number of the in-plane $\mathrm{O} 2$ stretching mode $B_{2 g}(1)$, being sensitive to the average $\mathrm{Mn}-\mathrm{O} 2$ bond length, does not allow us to follow separately the long $(l)$ and short $(s) \mathrm{Mn}-\mathrm{O} 2$ bond lengths, or the difference $l-s$, which can be seen as a simplified measure of the Jahn-Teller distortion. In previous studies, however, the characteristic signature of the reduction of Jahn-Teller effect in $\mathrm{LaMnO}_{3}$ and related compounds was the splitting of the $B_{2 g}(1)$ stretching mode at a high wave number and a gradual intensity transfer from one component to another $[5,10]$. This intensity transfer was assigned to the formation of "domains" of distorted and regular octahedra, observed from 3 to $34 \mathrm{GPa}$, and the gradual disappearance of the former. Here, our spectra at ambient conditions (Fig. 1) do show a small peak appearing as a shoulder of the $B_{2 g}(1)$ mode, and it can be followed with pressure (Fig. 3), but we do not observe any intensity transfer for any of the compounds, including $\mathrm{PrMnO}_{3}$. This was also the case in $\mathrm{BiMnO}_{3}$ [24]. This suggests that, in our experiments, if coexistence of distorted and undistorted octahedra actually takes place, it is not significantly affected by pressure. Since diffraction gives nonetheless clear evidence for a reduction of Jahn-Teller distortion, at least at low pressures, this can be interpreted by a homogeneous reduction of the Jahn-Teller distortion throughout the sample. This point, however, needs to be clarified by further studies.

\section{Hydrostatic pressure versus chemical pressure}

We now discuss our high-pressure data in the light of the concept of chemical pressure in rare-earth manganites. Generally speaking, in a solid solution, the substitution of a rare-earth cation on the $A$ site by a smaller isovalent cation causes a reduction of cell volume and is therefore depicted as a positive (compressive) chemical pressure. Because the substituted cations are supposed to be distributed in a random and homogeneous way, which is itself an assumption that could possibly be challenged, this chemical pressure is assumed isotropic, i.e. implicitly compared to hydrostatic pressure. For the case of rare-earth manganites, we know indeed that the volume depends linearly on the ionic radius [2], and the lattice parameters can be expected to follow Vegard's law [38]. If the analogy between hydrostatic and chemical pressures were perfectly valid, then the lattice parameters and other physical properties of a given manganite should just depend on its volume, irrespective of whether this volume is reached by chemical substitution or hydrostatic pressure. Our experimental data enable us to examine the validity of this analogy by plotting physical quantities as a function of volume across the full $\mathrm{AMnO}_{3}$ series.

Figure 8 shows the pseudocubic lattice parameters of orthorhombic rare-earth manganites as a function of $V_{p c}{ }^{1 / 3}$ for different rare-earth manganites at room conditions [39-43]. It shows that the chemical pressure does not act in the same way in the three lattice parameters of the rare-earth manganites. While pseudocubic lattice parameters $b_{p c}$ and $c_{p c}$ decrease monotonously with decreasing ionic radius from $\mathrm{La}^{3+}$ to $\mathrm{Lu}^{3+}, a_{p c}$ shows a nonmonotonous behavior, with a maximum located around Eu. On the same plot, we show a selection of data measured under hydrostatic pressures, having in mind that the lattice parameters of a given solid solution under the effect of chemical pressure are expected to lie on a straight line between the two end compounds. For the larger $A$ cation, a discrepancy is immediately apparent: the lattice parameters under hydrostatic pressure for $\mathrm{LaMnO}_{3}$ do not follow, at all, 

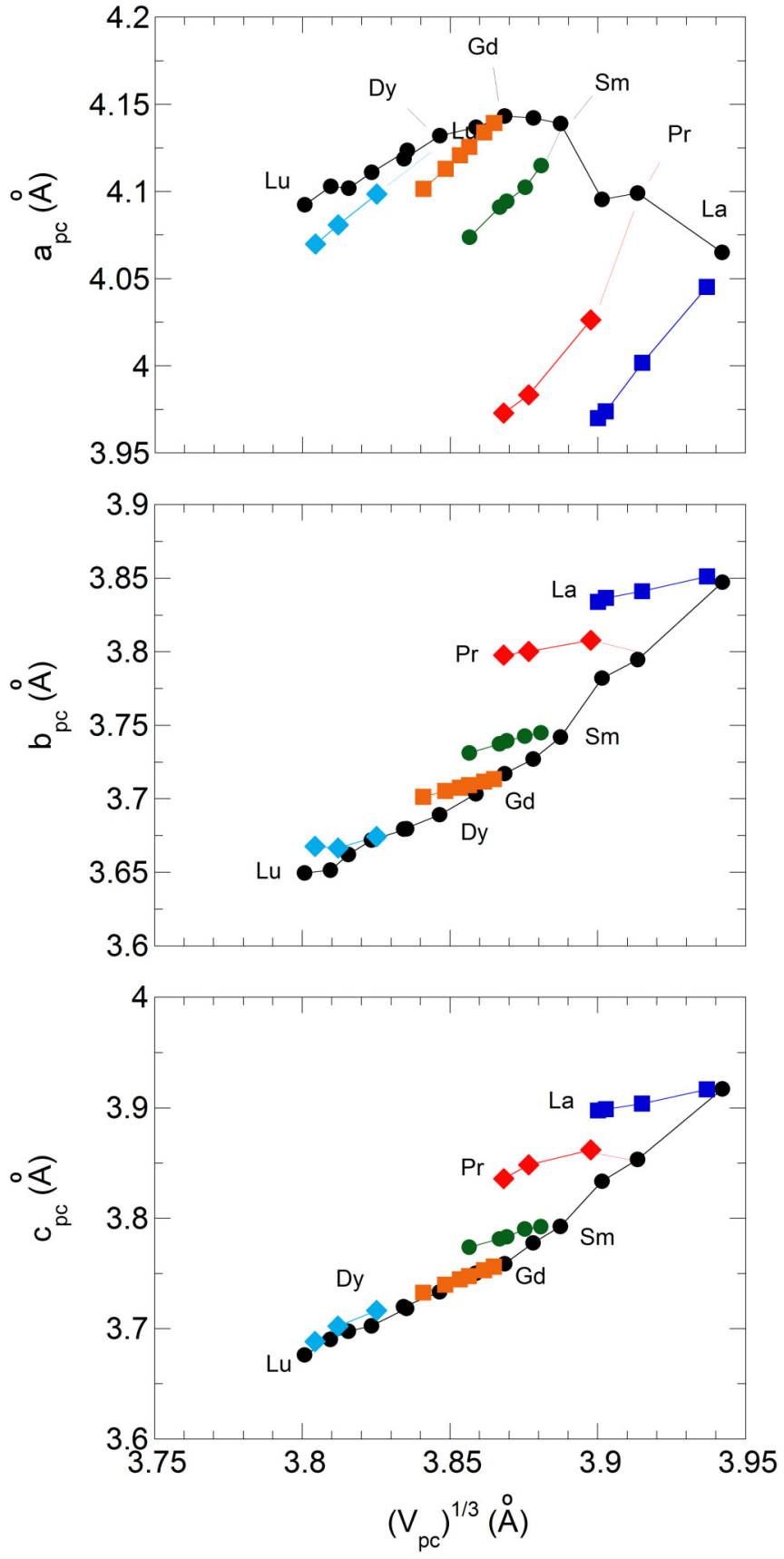

FIG. 8. (Color online) Pseudocubic lattice parameters of some orthorhombic rare-earth manganites, as a function of $V_{p c}{ }^{1 / 3}$, measured at room conditions and at different fixed hydrostatic pressures.

the La-Pr line, the most striking case being $a_{p c}$ which decreases under hydrostatic pressure whereas it increases under chemical pressure. For the smallest cations measured here (Dy), the hydrostatic pressure evolution of the $b_{p c}$ and $c_{p c}$ does follow more closely the line of cation substitution, although even in that case, the match is not perfect. The analogy between hydrostatic pressure and chemical pressure therefore appears reasonable for small cations but very misleading for larger cations. Similar conclusions could be drawn by plotting, for example, the Raman wave numbers as a function of volume.

This difference between chemical pressure and hydrostatic pressure merely reflects the diversity and complexity of the compression mechanisms at work in the manganites. The analogy is valid when compression is isotropic and realized through a homogeneous shortening of all chemical bonds, which is approximately realized in manganites with small $A$ cations (Dy to Lu). Conversely, the analogy clearly fails for manganites with larger cations because other compression mechanisms come into play (Jahn-Teller distortion and tilts).

\section{CONCLUSIONS}

In this paper, we report a high-pressure Raman scattering study in orthorhombic rare-earth manganites $\mathrm{AMnO}_{3}$, with $A=\mathrm{Pr}, \mathrm{Nd}, \mathrm{Sm}, \mathrm{Eu}, \mathrm{Tb}$, and $\mathrm{Dy}$, and synchrotron XRD in $A=\mathrm{Pr}, \mathrm{Sm}, \mathrm{Eu}$, and Dy. Our data, together with a review of previous studies, provide an overview of the behavior of manganites under hydrostatic pressure. The general picture shows a gradual evolution across the rare-earth series between two different behaviors.

For small rare-earth cations, the compression under hydrostatic pressure is close to isotropic in the $a c$ plane, with only small evolutions of the tilt angles and CJTD, as revealed by the evolution of the shear strain. The bulk modulus $(\approx 170 \mathrm{GPa})$ and the transition pressure $(\approx 48 \mathrm{GPa})$ are nearly independent of the rare-earth cation, and the effect of chemical pressure and hydrostatic pressure are similar. On the other hand, for large rare-earth cations, the reduction of the CJTD contributes significantly to the volume reduction. The bulk modulus and the transition pressure decrease strongly as the ionic radius increases. The anomaly of the shear spontaneous strain suggests a suppression of the average CJTD before the phase transition is reached. Chemical substitution and hydrostatic pressure have distinctly different effects.

In all cases, Raman spectroscopy gives evidence for a phase transition at high pressure. For the compounds with $A=\mathrm{Pr}, \mathrm{Sm}$, and Dy, XRD confirms the presence of a corresponding structural transition to a noncubic phase, so that the disappearance of the Raman spectrum can be interpreted as an insulator-to-metal transition. Further structural studies are needed in order to confirm this scenario for the other compounds. The crystal structures of the high-pressure phases vary from compound to compound, and no general rule emerges so far. This variety, however, could be understood if the different compression behaviors described above lead to different mechanisms at the phase transition.

Still, this schematic overview needs to be confirmed, and several aspects call for future work: the detailed crystal structures of the high-pressure phases, the possibly different mechanisms of the insulator-to-metal transition for the different rare-earth manganites, the process by which Jahn-Teller distortion is reduced by pressure (with or without coexistence of distorted and undistorted octahedra), and the associated Raman signature. Single crystal studies would be necessary to rule out spurious effects resulting from extrinsic shear stresses developing between grains in a powder. Further studies are therefore needed for a thorough understanding of the behavior of rare-earth manganites under hydrostatic pressure.

\section{ACKNOWLEDGMENTS}

This work was supported by Fundação para a Ciência e a Tecnologia through the Project 
PTDC/FIS-NAN/0533/2012, by Quadro de Referência Estratégica Nacional through Projeto Norte-070124FEDER-000070 Nanomateriais Multifuncionais, by Fundo Europeu de Desenvolvimento Regional through the COMPETE Program (Programa Operacional Factores de Competitividade), and by the Portuguese Foundation for Science and Technology within the strategic Project
PEST-C/FIS/UI0036/2011, and by the National Research Fund, Luxembourg (Contract No. FNR/P12/4853155/Kreisel). We acknowledge DESY PETRA-Grant III in Hamburg, Germany, and ESRF in Grenoble, France for providing beam time. We also acknowledge Zuzana Konopkova (DESY), Hans-Peter Liermann (DESY), and Gaston Garbarino (ESRF) for their support during the experiments.
[1] R. H. Mitchell, Perovskites, Modern and Ancient (Almaz Press, Ontario, Canada, 2002).

[2] J. A. Alonso, M. J. Martínez-Lope, M. T. Casais, and M. T. Fernández-Díaz, Inorg. Chem. 39, 917 (2000).

[3] M. Mochizuki and N. Furukawa, Phys. Rev. B 80, 134416 (2009).

[4] M. Mochizuki, N. Furukawa, and N. Nagaosa, Phys. Rev. B 84, 144409 (2011).

[5] I. Loa, P. Adler, A. Grzechnik, K. Syassen, U. Schwarz, M. Handfland, G. Kh. Rozenberg, P. Gorodetsky, and M. P. Pasternak, Phys. Rev Lett. 87, 125501 (2001).

[6] G. Trimarchi and N. Binggeli, Phys Rev. B 71, 035101 (2005).

[7] A. Yamasaki, M. Feldbacher, Y.-F. Yang, O. K. Andersen, and K. Held, Phys. Rev. Lett. 96, 166401 (2006).

[8] Javier D. Fuhr, Michel Avignon, and Blas Alascio, Phys. Rev. Lett. 100, 216402 (2008).

[9] J. He, M.-X. Chen, X.-Q. Chen, and C. Franchini, Phys. Rev. B 85, 195135 (2012).

[10] M. Baldini, V. V. Struzhkin, A. F. Goncharov, P. Postorino, and W. L. Mao, Phys. Rev. Lett. 106, 066402 (2011).

[11] A. Y. Ramos, N. M. Souza-Neto, H. C. N. Tolentino, O. Bunau, Y. Joly, S. Grenier, J.-P. Itié, A.-M. Flank, P. Lagarde, and A. Caneiro, Europhys. Lett. 96, 36002 (2011).

[12] J. M. Chen, T. L. Chou, J. M. Lee, S. A. Chen, T. S. Chan, T. H. Chen, K. T. Lu, W. T. Chuang, H.-S. Sheu, S. W. Chen, C. M. Lin, N. Hiraoka, H. Ishii, K. D. Tsuei, and T. J. Yang, Phys. Rev. B 79, 165110 (2009).

[13] J. M. Chen, J. M. Lee, T. L. Chou, S. A. Chen, S. W. Huang, H. T. Jeng, K. T. Lu, T. H. Chen, Y. C. Liang, S. W. Chen, W. T. Chuang, H.-S. Sheu, N. Hiraoka, H. Ishii, K. D. Tsuei, Eugene Huang, C. M. Lin, and T. J. Yang, J. Chem. Phys. 133, 154510 (2010).

[14] L. Martín-Carrón, J. Sánchez-Benítez, and A. de Andrés, J. Solid State Chem. 171, 313 (2003).

[15] J. Oliveira, J. Agostinho Moreira, A. Almeida, V. H. Rodrigues, M. M. R. Costa, P. B. Tavares, P. Bouvier, M. Guennou, and J. Kreisel, Phys. Rev. B 85, 052101 (2012).

[16] D. V. S. Muthu, A. E. Midgley, P. R. Scott, M. B. Kruger, J. R. Sahu, A. K. Sood, and C. N. R. Rao, J. Physics: Conference Series 377, 012025 (2012).

[17] J. Agostinho Moreira, A. Almeida, W. S. Ferreira, M. R. Chaves, J. B. Oliveira, J. M. Machado da Silva, M. A. Sá, S. M. F. Vilela, and P. B. Tavares, J. Electroceram. 25, 203 (2010).

[18] A. Dewaele and P. Loubeyre, High Press. Res. 27, 419 (2007).

[19] K. Takemura and A. Dewaele, Phys. Rev. B 78, 104119 (2008).

[20] H. K. Mao, J. Xu, and P. M. Bell, J. Geophys. Res.: Solid Earth 91, 4673 (1986)
[21] A. P. Hammersley, S. O. Svensson, M. Hanfland, A. N. Fitch, and D. Hausermann, High Pressure Res. 14, 235 (1996).

[22] J. Rodriguez-Carvajal, Physica B 192, 55 (1993).

[23] G. Kh. Rozemberg, M. P. Pasternak, W. M. Xu, L. S. Dubrovinsky, S. Carlson, and R. D. Taylor, Europhys. Lett. 71, 228 (2005).

[24] M. Guennou, P. Bouvier, P. Toulemonde, C. Darie, C. Goujon, P. Bordet, M. Hanfland, and J. Kreisel, Phys. Rev. Lett. 112, 075501 (2014).

[25] F. Birch, Phys. Rev. 71, 809 (1947).

[26] V. S. Bhadram, D. Swain, R. Dhanya, A. Sundaresan, C. Narayana, arXiv:1312.6778.

[27] J. Laverdière, S. Jandl, A. A. Mukhin, V. Yu. Ivanov, V. G. Ivanov, and M. N. Iliev, Phys. Rev. B 73, 214301 (2006).

[28] M. N. Iliev, M. V. Abrashev, J. Laverdière, S. Jandl, M. M. Gospodinov, Y.-Q. Wang, and Y.-Y. Sun, Phys. Rev. B 73, 064302 (2006).

[29] N. D. Todorov, M. V. Abrashev, and V. G. Ivanov, J. Phys.: Condens. Matter 24, 175404 (2012).

[30] See Supplemental Material at http://link.aps.org/supplemental/ 10.1103/PhysRevB.90.054104 for the pressure dependence of the Raman wave number determined from the fits to the spectra, for the compounds $\mathrm{AMnO}_{3}$, with $\mathrm{A}=\mathrm{Pr}, \mathrm{Sm}, \mathrm{Nd}, \mathrm{Eu}, \mathrm{Tb}$, and Dy; mode assignment according to [28].

[31] J. F. Vetelino, S. S. Mitra, and K. V. Namjoshi, Phys. Rev. B 2, 2167 (1970).

[32] N. L. Vočadlo and G. D. Price, Phys. Earth Planet. Inter. 82, 261 (1994).

[33] R. J. Angel, Rev. in Mineral. 39, 85 (2000).

[34] R. J. Angel, EoSFit V5.2 Users Manual. Ross Angel Software. 2011.

[35] M. W. Lufaso and P. M. Woodward, Acta Cryst. B 60, 10 (2004).

[36] M. A. Carpenter and C. J. Howard, Acta Cryst. B 65, 134 (2009).

[37] M. A. Carpenter and C. J. Howard, Acta Cryst. B 65, 147 (2009).

[38] A. R. Denton and N. W. Ashcroft, Phys. Rev. A 43, 3161 (1991).

[39] L. Pinsard-Gaudart, J. Rodríguez-Carvajal, A. Daoud-Aladine, I. Goncharenko, M. Medarde, R. I. Smith, and A. Revcolevschi, Phys. Rev. B 64, 064426 (2001).

[40] T. Mori, N. Kamegashira, K. Aoki, T. Shishido, and T. Fukuda, Mat. Lett. 54, 238 (2002).

[41] K. Uusi-Esko, J. Malmb, N. Imamuraa, H. Yamauchia, and M. Karppinen, Mat. Chem. Phys. 112, 1029 (2008).

[42] Y. Chen, H. Yuan, G. Li, Ge Tian, and S. Feng, J. Cryst. Growth 305, 242 (2007).

[43] J.-S. Zhou, J. B. Goodenough, J. M. Gallardo-Amores, E. Morán, M. A. Alario-Franco, and R. Caudillo, Phys. Rev. B 74, 014422 (2006). 